\title{
A class of solvable non-homogeneous differential operators on the Heisenberg group
}

\author{
by \\ Detlef Müller (Kiel) and Zhenqiu Zhang (Tainjin)
}

\begin{abstract}
In [8], we studied the problem of local solvability of complex coefficient second order left-invariant differential operators on the Heisenberg group $\mathbb{H}_{n}$, whose principal parts are "positive combinations of generalized and degenerate generalized subLaplacians", and which are homogeneous under the Heisenberg dilations. In this note, we shall consider the same class of operators, but in the presence of left invariant lower order terms, and shall discuss local solvability for these operators in a complete way. Previously known methods to study such non-homogeneous operators, as in [9] or [6], do not apply to these operators, and it is the main purpose of this article to introduce a new method, which should be applicable also in much wider settings.
\end{abstract}

1. Basic definitions and main results. Let $\omega$ denote the symplectic form on $\mathbb{R}^{2 n}$ given by

$$
\omega\left(z, z^{\prime}\right):={ }^{\mathrm{t}} z^{\prime} J z, \quad J=J_{n}:=\left(\begin{array}{cc}
0 & I_{n} \\
-I_{n} & 0
\end{array}\right) .
$$

The Heisenberg group $\mathbb{H}_{n}$ is $\mathbb{R}^{2 n} \times \mathbb{R}$, endowed with the group law

$$
(z, u)\left(z^{\prime}, u\right)=\left(z+z^{\prime}, u+u^{\prime}-\frac{1}{2} \omega\left(z, z^{\prime}\right)\right) .
$$

The left-invariant vector fields

$$
X_{j}:=\frac{\partial}{\partial x_{j}}-\frac{1}{2} y_{j} \frac{\partial}{\partial u}, \quad Y_{j}:=\frac{\partial}{\partial y_{j}}+\frac{1}{2} x_{j} \frac{\partial}{\partial u},
$$

$j=1, \ldots, n$, and $U:=\partial / \partial u$ form a natural basis for the Lie algebra $\mathfrak{h}_{n}$ of $\mathbb{H}_{n}$. The only non-trivial commutation relations among these vector fields are $\left[X_{j}, Y_{j}\right]=U, j=1, \ldots, n$.

Denote by $\mathfrak{s p}(n, \mathbb{C})$ the symplectic Lie algebra, consisting of all complex $2 n \times 2 n$-matrices $S$ satisfying

$$
{ }^{\mathrm{t}} S J+J S=0 .
$$

2000 Mathematics Subject Classification: 35A05, 43A80.

Work of the second author is supported by NNSF of China and NSF of Tianjin University. 
Let $S \in \mathfrak{s p}(n, \mathbb{C})$, denote by $A=\left(a_{j k}\right)$ the symmetric matrix $A:=S J$, and put

$$
\Delta_{S}:=\sum_{j, k=1}^{2 n} a_{j k} V_{j} V_{k},
$$

where $V_{j}:=X_{j}, V_{n+j}:=Y_{j}, j=1, \ldots, n$.

In [5], the situation where the matrix $S$ assumes a block diagonal form

$$
S=\left(\begin{array}{ccc}
\gamma_{1} S_{(1)} & & \\
& \ddots & \\
& & \gamma_{m} S_{(m)}
\end{array}\right)
$$

with respect to a suitable decomposition of $\mathbb{R}^{2 n}$ into symplectic subspaces has been studied, under the assumptions that $\gamma_{j} \in \mathbb{C}^{\times}=\mathbb{C} \backslash\{0\}$ and $S_{(j)}^{2}=-I, j=1, \ldots, m$. By means of Hörmander's criterion, it has been shown that for "most" of these matrices $S$, the operators $\Delta_{S}+$ lower order terms are locally non-solvable.

There are only five exceptional classes of operators of the above type to which Hörmander's criterion does not apply and which are listed in [5]. In all these classes, each of the elementary blocks is of size $2 \times 2$. Moreover, according to the classification of normal forms in [7], after applying a suitable symplectic change of coordinates, one may assume that $S_{(j)}$ is either of the form

$$
S_{(j)}=\left(\begin{array}{cc}
i \varepsilon_{j} \lambda_{j} & \lambda_{j}^{2}-1 \\
1 & -i \varepsilon_{j} \lambda_{j}
\end{array}\right), \quad \text { "Type } 1 ",
$$

with $\lambda_{j} \in\{-1\} \cup\left[0, \infty\left[\right.\right.$ and $\varepsilon_{j}=1$ if $\left|\lambda_{j}\right| \leq 1$, and $\varepsilon_{j}= \pm 1$ if $\lambda_{j}>1$, or of the form

$$
S_{(j)}=\left(\begin{array}{cc}
0 & i \\
i & 0
\end{array}\right), \quad \text { "Type } 3 " .
$$

Denote by $\sigma_{S}$ the principal symbol of $-\Delta_{S}$, and assume henceforth that $\operatorname{Re} \sigma_{S} \geq 0$. Then $\Delta_{S}$ belongs to one of the major exceptional classes listed in [5]. It follows from [5] that $\Delta_{S}$ is a positive combination of generalized sub-Laplacians and of degenerate generalized sub-Laplacians, i.e.

$$
\begin{aligned}
\Delta_{S}= & \sum_{j=1}^{r} \gamma_{j}\left[\left(1-\lambda_{j}^{2}\right) X_{j}^{2}+Y_{j}^{2}+i \lambda_{j}\left(X_{j} Y_{j}+Y_{j} X_{j}\right)\right] \\
& +i \sum_{j=r+1}^{n} \gamma_{j}\left(X_{j}^{2}-Y_{j}^{2}\right)
\end{aligned}
$$

where $0 \leq r \leq n,\left|\lambda_{j}\right| \leq 1, \gamma_{j} \in \mathbb{C}^{\times}$for $j=1, \ldots, r, \gamma_{j}>0$ for $j=$ $r+1, \ldots, n$, and where for each $j=1, \ldots, r$ and every $\xi_{j}, \eta_{j} \in \mathbb{R}$, 


$$
\operatorname{Re}\left[\gamma_{j}\left[\left(1-\lambda_{j}^{2}\right) \xi_{j}^{2}+\eta_{j}^{2}+2 i \lambda_{j} \xi_{j} \eta_{j}\right]\right] \geq 0
$$

provided we choose appropriate coordinates.

In this article, we deal with the operators of the form

$$
L_{\alpha}:=\Delta_{S}+\sum_{j=1}^{n}\left(\beta_{j 1} X_{j}+\beta_{j 2} Y_{j}\right)+\alpha U+c,
$$

where the coefficients $\beta_{j 1}, \beta_{j 2}, \alpha$ and $c$ are complex. We assume in addition that

$$
\operatorname{Re} \gamma_{j}>0 \quad \text { for } j=1, \ldots, r
$$

(for a discussion of this condition, see Remark 1.2 in [8]).

Set

$$
E^{ \pm}:=\left\{ \pm \sum_{j=1}^{r} \gamma_{j}\left(2 l_{j}+1\right): l_{1}, \ldots, l_{r} \in \mathbb{N}\right\} .
$$

Moreover, denote by $n_{1}, n_{2}^{+}$and $n_{2}^{-}$the number of "Type 1 " blocks $S_{(j)}$ in $S$ with $\left|\lambda_{j}\right|<1, \lambda_{j}=1$ and $\lambda_{j}=-1$, respectively, and by $n_{3}$ the number of "Type 3" blocks.

Since $S_{(j)}^{2}=-I$ and $\gamma_{j} \neq 0, S$ is invertible, and so is the coefficient matrix $A$. We choose coordinates $z:=\left(z_{1}, \ldots, z_{n}\right)$, with $z_{j}:=\left(x_{j}, y_{j}\right)$, corresponding to the block form (1.3) of $S$. With respect to these coordinates, the matrix $A$ also assumes a block form

$$
A=\left(\begin{array}{ccc}
A_{(1)} & & \\
& \ddots & \\
& & A_{(n)}
\end{array}\right) .
$$

Thus, arguing as in [5], we see that it is possible to eliminate the first order terms in the $X_{j}$ 's and $Y_{j}$ 's, by conjugating $L_{\alpha}$ by a multiplication operator. In fact

$$
\begin{aligned}
& L_{\alpha}\left(e^{-(1 / 2)^{\mathrm{t}} z A^{-1} \beta} f\right) \\
& \quad=e^{-(1 / 2)^{\mathrm{t}} z A^{-1} \beta}\left(\Delta_{S}+i \alpha U+c-\frac{1}{4} \sum_{j=1}^{n}{ }^{\mathrm{t}} \beta_{j} A_{(j)}^{-1} \beta_{j}\right) f,
\end{aligned}
$$

where ${ }^{\mathrm{t}} \beta_{j}:=\left(\beta_{j 1}, \beta_{j 2}\right)$ and ${ }^{\mathrm{t}} \beta:=\left({ }^{\mathrm{t}} \beta_{1}, \ldots,{ }^{\mathrm{t}} \beta_{n}\right)$. Combining this observation with the results in $[8]$, we obtain

Theorem 1.1. Suppose $L_{\alpha}$, given by (1.6) and (1.8), satisfies (1.7) and (1.9), and assume that $c=\frac{1}{4} \sum_{j=1}^{n}{ }^{\mathrm{t}} \beta_{j} A_{(j)}^{-1} \beta_{j}$. Then the following hold:

(I) If $\alpha \notin E^{+} \cup E^{-}$, then $L_{\alpha}$ is locally solvable.

(II) If $\alpha \in E^{-}$, then $L_{\alpha}$ is locally solvable if and only if $n_{2}^{+}+n_{3}>0$.

(III) If $\alpha \in E^{+}$, then $L_{\alpha}$ is locally solvable if and only if $n_{2}^{-}+n_{3}>0$. 
This theorem discusses local solvability of those operators which can be reduced to homogeneous ones. For the remaining cases, we shall prove the following positive result.

Theorem 1.2. Suppose $L_{\alpha}$, given by (1.6) and (1.8), satisfies (1.7) and (1.9), and that $c \neq \frac{1}{4} \sum_{j=1}^{n}{ }^{\mathrm{t}} \beta_{j} A_{(j)}^{-1} \beta_{j}$. Then $L_{\alpha}$ is locally solvable.

REMARK 1.3. In Theorem 3.3 of [5], second order left-invariant differential operators on the Heisenberg group $\mathbb{H}_{1}$ were studied in a rather complete way. Following the notation of Theorem 3.3 in [5], the only case which was left open was the case where $\Delta_{S}$ is of type (a.2) and $c \neq\left(\beta_{1}^{2}-2 i \varepsilon \beta_{1} \beta_{2}\right) / 4$. Our main result shows that in this case the operator $L$ is locally solvable.

2. Proof of Theorem 1.2. We apply the usual convention that $C$ is a constant whose value may change from line to line.

In view of (1.10), we may assume that $\beta=0$, i.e. that $L_{\alpha}=\Delta_{S}+i \alpha U+c$, where $c \neq 0$. Denote by $f^{\mu}$ the partial Fourier transform of $f$ along the center of $\mathbb{H}_{n}$, i.e.

$$
f^{\mu}(z):=\int_{\mathbb{R}} f(z, u) e^{-i \mu u} d u, \quad \mu \in \mathbb{R}^{\times} .
$$

Moreover, for suitable functions or distributions $\varphi, \psi$ on $\mathbb{R}^{2 n}$, define the $\mu$-twisted convolution of $\varphi$ and $\psi$ by

$$
\varphi \times_{\mu} \psi(z):=\int_{\mathbb{R}^{2 n}} \varphi\left(z-z^{\prime}\right) \psi\left(z^{\prime}\right) e^{i(\mu / 2) \omega\left(z-z^{\prime}, z^{\prime}\right)} d z^{\prime} .
$$

Then one easily verifies that, for suitable distributions $f, g$ on $\mathbb{H}_{n}$,

$$
(f * g)^{\mu}=f^{\mu} \times_{\mu} g^{\mu} .
$$

Denote by $\mathfrak{u}\left(\mathfrak{h}_{n}\right)$ the universal enveloping algebra of $\mathfrak{h}_{n}$. Then $\mathfrak{u}\left(\mathfrak{h}_{n}\right)$ can be identified with the associative algebra of all left-invariant differential operators on $\mathbb{H}_{n}$. If $D \in \mathfrak{u}\left(\mathfrak{h}_{n}\right)$, we define the partial Fourier transform of $D$ as the partial differential operator $D^{\mu}$ on $\mathbb{R}^{2 n}$ which is given by the formula

$$
D^{\mu} f^{\mu}=(D f)^{\mu}, \quad f \in S\left(\mathbb{H}_{n}\right),
$$

where $S\left(\mathbb{H}_{n}\right)$ denotes the Schwartz space on $\mathbb{H}_{n}$. In particular, we then have $L_{\alpha}^{\mu}=\Delta_{S}^{\mu}-\alpha \mu+c$.

Let $f \in S\left(\mathbb{H}_{n}\right)$. We intend to solve the equation

$$
L_{\alpha} v=f .
$$

Proceeding formally as in [5], [8], we put

$$
\Gamma_{t, S}^{\mu}(z):=\frac{|\mu|^{n}}{(4 \pi)^{n} \prod_{j=1}^{n} \sinh \left(\gamma_{j} t\right)} e^{-(|\mu| / 4) \sum_{j=1}^{n} \operatorname{coth}\left(\gamma_{j} t\right)^{\mathrm{t}} z_{j} J_{1} S_{(j)} z_{j}}, \quad \mu \in \mathbb{R}^{\times} .
$$


From [5], we have

$$
|\mu| \frac{d}{d t}\left(\varphi \times_{\mu} \Gamma_{t, S}^{\mu}\right)=\Delta_{S}^{\mu}\left(\varphi \times_{\mu} \Gamma_{t, S}^{\mu}\right)
$$

for every $\varphi \in S\left(\mathbb{R}^{2 n}\right)$. Therefore

$$
\begin{aligned}
|\mu| \frac{d}{d t}\left[e ^ { - \alpha ( \operatorname { s g n } \mu ) t + ( c / | \mu | ) t } \left(\varphi \times{ }_{\mu}\right.\right. & \left.\left.\Gamma_{t, S}^{\mu}\right)\right] \\
& =L_{\alpha}^{\mu}\left[e^{-\alpha(\operatorname{sgn} \mu) t+(c /|\mu|) t}\left(\varphi \times{ }_{\mu} \Gamma_{t, S}^{\mu}\right)\right] .
\end{aligned}
$$

So a fundamental solution for $L_{\alpha}=\Delta_{S}+i \alpha U+c$ can be formally defined by

$$
F_{\alpha}(z, u):=-\frac{1}{2 \pi} \int_{0}^{\infty} \int_{\mathbb{R}^{\times}} e^{-\alpha(\operatorname{sgn} \mu) t+(c /|\mu|) t} \Gamma_{t, S}^{\mu}(z) e^{i \mu u} \frac{d \mu}{|\mu|} d t .
$$

On a formal level, this suggests that a solution to the equation $L_{\alpha} v=f$ is given by

$$
\begin{aligned}
v(z, u) & =f * F_{\alpha}(z, u) \\
& =-\frac{1}{2 \pi} \int_{0}^{\infty} \int_{\mathbb{R}^{\times}} e^{-a(\operatorname{sgn} \mu) t+(c /|\mu|) t} f^{\mu} \times_{\mu} \Gamma_{t, S}^{\mu}(z) e^{i \mu u} \frac{d \mu}{|\mu|} d t .
\end{aligned}
$$

However, the integral (2.3) will in general not be convergent. A main and principal obstacle is the exponential growth of the factor $e^{(c /|\mu|) t}$ as $\mu \rightarrow 0$, in the case where $\operatorname{Re} c>0$.

On the other hand, in order to prove local solvability, we do not really have to construct a fundamental solution, and are even allowed to ignore "low frequencies" (compare also [1]), as the following auxiliary result shows.

Fix a cut-off function $\chi \in C_{0}^{\infty}\left(\mathbb{R}^{d}\right)$ such that $0 \leq \chi \leq 1, \chi(x)=1$ for $|x| \leq 1$ and $\chi(x)=0$ for $|x|>2$, and write $\chi_{B}(x):=\chi(x / B)$ if $B>0$. We introduce Fourier multiplier operators $Q_{B}$ and $P_{B}$ on $L^{2}\left(\mathbb{R}^{d}\right)$ by

$$
\widehat{Q_{B} f}:=\chi_{B} \widehat{f}, \quad P_{B} f=\left(I-Q_{B}\right) f, \quad f \in L^{2}\left(\mathbb{R}^{d}\right) .
$$

Proposition 2.1. There exists a constant $K>1$, depending only on the dimension $d$, such that the following holds: If $r$ and $B$ are positive numbers such that $r<1 /(K B)$, then for every function $f \in L^{2}\left(\mathbb{R}^{d}\right)$ which is supported in the ball $B_{r}(0):=\left\{x \in \mathbb{R}^{d}:|x|<r\right\}$, we have

$$
\|f\|_{2} \leq 2\left\|\chi_{2 r}\left(P_{B} f\right)\right\|_{2} .
$$

Proof. Denote by $\eta_{B}$ the inverse Fourier transform of $\chi_{B}$. Then for every $N>0$ we find a constant $C=C_{N}>0$ such that

$$
\left|\eta_{B}(x)\right| \leq C B^{d}(1+|B x|)^{-N} \text {. }
$$


Assume that $f \in L^{2}\left(\mathbb{R}^{d}\right)$ and $\operatorname{supp} f \subset B_{r}(0)$. If $|x| \geq 2 r$, then

$$
\begin{aligned}
\left|\left(P_{B} f\right)(x)\right| & =\left|f(x)-\eta_{B} * f(x)\right|=\left|\eta_{B} * f(x)\right|=\left|\int_{|y| \leq r} f(y) \eta_{B}(x-y) d y\right| \\
& \leq C B^{d}\|f\|_{2}\left(\int_{|y| \leq r}(1+B|x-y|)^{-2 N} d y\right)^{1 / 2} \\
& \leq C B^{d} r^{d / 2}\|f\|_{2}(1+B|x|)^{-N} .
\end{aligned}
$$

Thus, if $r<1 /(K B)$, and if we choose $N$ such that $d>N>d / 2$, we obtain

$$
\begin{aligned}
\left(\int_{|x| \geq 2 r}\left|\left(P_{B} f\right)(x)\right|^{2} d x\right)^{1 / 2} & \leq C B^{d} r^{d / 2}\|f\|_{2}\left(\int_{|x| \geq 2 r}|B x|^{-2 N} d x\right)^{1 / 2} \\
& =C B^{d-N} r^{d-N}\|f\|_{2} \leq C K^{-(d-N)}\|f\|_{2} .
\end{aligned}
$$

On the other hand, since $f=\chi_{r} f$, we have

$$
|\widehat{f}(\xi)|=\left|\widehat{\chi}_{r} * \widehat{f}(\xi)\right| \leq\|\widehat{f}\|_{2} \cdot\left\|\widehat{\chi}_{r}\right\|_{2} \leq C r^{d / 2}\|f\|_{2}
$$

for every $\xi \in \mathbb{R}^{d}$. Therefore, by Plancherel's formula,

$$
\left\|Q_{B} f\right\|_{2} \leq\left(\int_{|\xi| \leq 2 B}|\widehat{f}(\xi)|^{2} d \xi\right)^{1 / 2} \leq C B^{d / 2} r^{d / 2}\|f\|_{2} \leq C K^{-d / 2}\|f\|_{2} .
$$

Choosing $K$ sufficiently large, we thus obtain

$$
\|f\|_{2} \leq\left\|Q_{B} f\right\|_{2}+\left\|P_{B} f\right\|_{2} \leq \frac{1}{2}\|f\|_{2}+\left\|\chi_{2 r}\left(P_{B} f\right)\right\|_{2} .
$$

This implies Proposition 2.1.

We shall apply the proposition above to the central variable $u$ on the Heisenberg group. To this end, we define for $B>0$ the operator $\mathcal{P}_{B}$ on $L^{2}\left(\mathbb{H}_{n}\right)$ by means of the formula

$$
\left(\mathcal{P}_{B} f\right)^{\mu}:=\left[1-\chi_{B}(\mu)\right] f^{\mu}, \quad \mu \in \mathbb{R}^{\times}, f \in S\left(\mathbb{H}_{n}\right) .
$$

Because of the blow-up of the integrated function that appears in (2.3), we shall try to solve the equation $L_{\alpha} v=\mathcal{P}_{B} f$ instead of $L_{\alpha} v=f$. Consider the distribution $H_{\alpha}$, which is formally defined by

$$
\begin{aligned}
\left\langle H_{\alpha}, \varphi\right\rangle:=\frac{-1}{2 \pi} \int_{0}^{\infty} \int\left(1-\chi_{B}(\mu)\right) e^{-\alpha(\operatorname{sgn} \mu) t+(c /|\mu|) t}\left\langle\Gamma_{t, S}^{\mu}, \varphi^{-\mu}\right\rangle \frac{d \mu}{|\mu|} d t, & \varphi \in \mathcal{S}\left(\mathbb{H}_{n}\right) .
\end{aligned}
$$

Proposition 2.2. Let $A:=\sum_{j} \operatorname{Re} \gamma_{j}$. If $|\operatorname{Re} \alpha|+|\operatorname{Re} c| / B<A$, then $H_{\alpha}$ is a well-defined tempered distribution on $\mathbb{H}_{n}$, satisfying

$$
L_{\alpha}\left(f * H_{\alpha}\right)=\mathcal{P}_{B} f \quad \text { for every } f \in \mathcal{S}\left(\mathbb{H}_{n}\right) .
$$

Moreover, if $A>|\operatorname{Re} c| / B$, then $\left\{H_{\alpha}\right\}_{|\operatorname{Re} \alpha|<A-|\operatorname{Re} c| / B}$ is an analytic family of tempered distributions. 
Proof. We closely follow [5], §§7.3, 7.4. If we put $\check{g}(z):=g(-z)$, then

$$
\left\langle\Gamma_{t, S}^{\mu}, \varphi^{-\mu}\right\rangle=\frac{1}{(2 \pi)^{n}}\left\langle\widehat{\Gamma_{t, S}^{\mu}}, \widehat{\varphi^{-\mu}}\right\rangle,
$$

where $\widehat{\Gamma_{t, S}^{\mu}}$ is given by

$$
\widehat{\Gamma_{t, S}^{\mu}}(\zeta)=\frac{1}{\sigma(t)} e^{-\frac{1}{|\mu|} q_{t}(\zeta)}
$$

with

$$
\begin{aligned}
\sigma(t) & :=\prod_{j=1}^{n} \cosh \left(\gamma_{j} t\right) \\
q_{t}(\zeta) & :=\sum_{j=1}^{r} \tanh \left(\gamma_{j} t\right)\left[\left(1-\lambda_{j}^{2}\right) \xi_{j}^{2}+\eta_{j}^{2}+2 i \lambda_{j} \xi_{j} \eta_{j}\right]+i \sum_{j=r+1}^{n} \tanh \left(\gamma_{j} t\right)\left(\xi_{j}^{2}-\eta_{j}^{2}\right) \\
& =: \sum_{j=1}^{n} \tanh \left(\gamma_{j} t\right) q_{j}\left(\zeta_{j}\right)
\end{aligned}
$$

if $\zeta_{j}=\left(\xi_{j}, \eta_{j}\right)$. Since $\operatorname{Re} q_{t} \geq 0$ (see [5]), we thus have

$$
\left|e^{-\alpha(\operatorname{sgn} \mu) t+(c /|\mu|) t} \widehat{\Gamma_{t, S}^{\mu}}(\zeta)\right| \leq C e^{-(-|\operatorname{Re} \alpha|-|\operatorname{Re} c| /|\mu|+A) t},
$$

and consequently, for $|\mu| \geq B$,

$$
\begin{aligned}
& \int_{0}^{\infty}\left|e^{-\alpha(\operatorname{sgn} \mu) t+(c /|\mu|) t} \widehat{\Gamma_{t, S}^{\mu}}(\zeta)\right| d t \leq C \frac{|\mu|}{(A-|\operatorname{Re} \alpha|)|\mu|-|\operatorname{Re} c|} \\
& \quad \text { for every } \zeta \in \mathbb{R}^{2 n} .
\end{aligned}
$$

This shows that the integral defining $\left\langle H_{\alpha}, \varphi\right\rangle$ is absolutely convergent, and that

$$
\left|\left\langle H_{\alpha}, \varphi\right\rangle\right| \leq C \int_{|\mu| \geq B} \int_{\mathbb{R}^{2 n}}\left|\frac{\widehat{\check{\varphi}^{-\mu}}(\zeta)}{(A-|\operatorname{Re} \alpha|)|\mu|-|\operatorname{Re} c|}\right| d \zeta d \mu \leq\|\varphi\|_{\mathcal{S}}
$$

for some Schwartz norm $\|\cdot\|_{\mathcal{S}}$. Moreover, the mapping $\alpha \mapsto\left\langle H_{\alpha}, \varphi\right\rangle$ is analytic for $|\operatorname{Re} \alpha|<A-|\operatorname{Re} c| / B$. Finally, from (2.1) we obtain

$$
L_{\alpha}\left(f * H_{\alpha}\right)(z, u)=\frac{1}{2 \pi} \int\left(1-\chi_{B}(\mu)\right) f^{\mu}(z) e^{i \mu u} d \mu=\mathcal{P}_{B} f(z, u) .
$$

Fix now $B$ such that $b_{0}:=A-|\operatorname{Re} c| / B>0$, and put $\gamma:=\sum_{j} \gamma_{j}$, $a:=\min _{j} \operatorname{Re} \gamma_{j}$ and $b_{m}:=b_{0}+2 m a, m \in \mathbb{N}$, so that $b_{m} \rightarrow \infty$ as $m \rightarrow \infty$. In particular, the domains $\Sigma_{m}:=\left\{\alpha \in \mathbb{C}:|\operatorname{Re} \alpha|<b_{m}\right\}, m \in \mathbb{N}$, cover the complex plane.

The analytic family of distributions $H_{\alpha}$ will in general not extend from $\Sigma_{0}$ to a larger strip $\Sigma_{m}$. However, arguing as in [5], by means of integrations by parts in the $t$-variable, we can prove the following 
Proposition 2.3. Let $m \in \mathbb{N}$. Then there exist an analytic family of tempered distributions $\left\{H_{\alpha}^{m}\right\}_{\alpha \in \Sigma_{m}}$ on $\mathbb{H}_{n}$ and a family of non-trivial differential operators $Q_{\alpha}=\sum_{k} a_{k}(\alpha) U^{k}$ whose coefficients $a_{k}(\alpha)$ depend polynomially on $\alpha$, such that

$$
L_{\alpha}\left(f * H_{\alpha}^{m}\right)=Q_{\alpha} \mathcal{P}_{B} f \quad \text { for } f \in \mathcal{S}\left(\mathbb{H}_{n}\right), \alpha \in \Sigma_{m} .
$$

Proof. First re-write $H_{\alpha}$ for $\alpha \in \Sigma_{0}$. Let $\varepsilon(\mu):=\operatorname{sgn} \mu$. If $J=\left(j_{1}, \ldots\right.$ $\left.\ldots, j_{m}\right)$ is a multi-index of length $\ell(J)=m$ in $\{1, \ldots, n\}^{m}$, we set

$$
\varrho_{J}(\mu):=\prod_{k=0}^{m}\left(\varepsilon(\mu) \alpha+\gamma+\sum_{l \leq k} 2 \gamma_{j_{l}}-\frac{c}{|\mu|}\right)
$$

(the factor with $k=0$ is taken to be $(\varepsilon(\mu) \alpha+\gamma-c /|\mu|)$ ). Put $M_{j}:=\gamma_{j} q_{j}$, and

$$
M_{J}:=M_{j_{1}} \ldots M_{j_{m}}
$$

(where $M_{\emptyset}:=1$, with $\ell(\emptyset)=0$ ). Then, after $m$ integrations by parts following the lines in [5], we find that there are functions $\beta_{J}$ on $\{-1,+1\}$ for $\ell(J)<m$, and functions $K_{\mu, J}(t)$ for $\ell(J)=m$, such that

$$
\begin{aligned}
\left\langle H_{\alpha}, f\right\rangle= & \sum_{\ell(J)<m} \int \frac{\beta_{J}(\varepsilon(\mu))}{\varrho_{\widetilde{J}}(\mu)} M_{J}(0) f^{-\mu}(0) \frac{1-\chi_{B}(\mu)}{|\mu|^{\ell(J)+1}} d \mu \\
& +\sum_{\ell(J)=m} \int_{0}^{\infty} \int_{0}^{\infty} K_{\mu, J}(t)\left\langle e^{-q_{t} /|\mu|}, M_{J} \widehat{\tilde{f}^{-\mu}}\right\rangle \frac{1-\chi_{B}(\mu)}{|\mu|^{m+1}} d \mu d t,
\end{aligned}
$$

and

$$
K_{\mu, J}(t) \sim \frac{c_{J}}{\varrho_{\widetilde{J}}(\mu)} e^{-\left(\varepsilon(\mu) \alpha+\gamma+\sum_{l=1}^{m} 2 \gamma_{j_{l}}-c /|\mu|\right) t},
$$

where $\widetilde{J}$ is obtained from $J$ by deleting the last index $j_{m}$.

In order to extend the definition of $H_{\alpha}$ by means of (2.8) and (2.9) to $\alpha \in \Sigma_{m}$, we have to "remove" the zeros of the functions $\varrho_{\widetilde{J}}$. To this end, put

$$
h_{\alpha, J}(\mu):=\prod_{k=0}^{m}\left[\left(\left(\alpha+\gamma+\sum_{l \leq k} 2 \gamma_{j_{l}}\right) \mu-c\right)\left(\left(\alpha-\gamma-\sum_{l \leq k} 2 \gamma_{j_{l}}\right) \mu-c\right)\right]
$$

and

$$
Q_{\alpha}(i \mu):=\prod_{\ell(J) \leq m} h_{\alpha, \widetilde{J}}(\mu)
$$

Then $Q_{\alpha}$ is a non-trivial polynomial, since $c \neq 0$. Moreover, $Q_{\alpha}(i \mu) / \varrho_{\widetilde{J}}(\mu)$ is a smooth function away from $\mu=0$ which grows at most polynomially. We therefore define a tempered distribution $H_{\alpha}^{m}$ by the formula 


$$
\begin{aligned}
\left\langle H_{\alpha}^{m}, \varphi\right\rangle:=\frac{-1}{2 \pi} \int_{0}^{\infty} \int\left(1-\chi_{B}(\mu)\right) e^{-\alpha(\operatorname{sgn} \mu) t+(c /|\mu|) t} & \\
& \times\left\langle\Gamma_{t, S}^{\mu}, \varphi^{-\mu}\right\rangle Q_{\alpha}(i \mu) \frac{d \mu}{|\mu|} d t, \quad \varphi \in \mathcal{S}\left(\mathbb{H}_{n}\right) .
\end{aligned}
$$

Replacing $H_{\alpha}$ by $H_{\alpha}^{m}$ in the preceding argument, we obtain

$$
\begin{aligned}
& \left\langle H_{\alpha}^{m}, f\right\rangle=\sum_{\ell(J)<m} \int \frac{Q_{\alpha}(i \mu) \beta_{J}(\varepsilon(\mu))}{\varrho_{\widetilde{J}}(\mu)} M_{J}(0) f^{-\mu}(0) \frac{1-\chi_{B}(\mu)}{|\mu|^{\ell(J)+1}} d \mu \\
& +\sum_{\ell(J)=m} \int_{0}^{\infty} \int Q_{\alpha}(i \mu) K_{\mu, J}(t)\left\langle e^{-q_{t} /|\mu|}, M_{J} \widehat{\tilde{f}^{-\mu}}\right\rangle \frac{1-\chi_{B}(\mu)}{|\mu|^{m+1}} d \mu d t .
\end{aligned}
$$

From (2.9), one finds that the right-hand side of (2.11) converges absolutely even for $\alpha \in \Sigma_{m}$, and that it defines an analytic family of tempered distributions $H_{\alpha}^{m}$ for $\alpha \in \Sigma_{m}$. Moreover, if $\alpha \in \Sigma_{0}$, we may argue as in the proof of Proposition 2.2 in order to verify (2.7). Finally, since both sides of (2.7) are analytic in $\alpha \in \Sigma_{m}$, we see that, by analytic continuation, (2.7) remains valid for every $\alpha \in \Sigma_{m}$.

Now, in order to prove Theorem 1.2, if $\alpha \in \mathbb{C}$ we choose $m \in \mathbb{N}$ such that $\alpha \in \Sigma_{m}$, and then $H_{\alpha}^{m} \in \mathcal{S}^{\prime}\left(\mathbb{H}_{n}\right)$ and the bi-invariant differential $Q_{\alpha}$ according to Proposition 2.3. Let $\mathcal{H}_{\alpha} f:=f * H_{\alpha}^{m}$ for $f \in \mathcal{S}\left(\mathbb{H}_{n}\right)$.

If we denote by ${ }^{\mathrm{t}} L_{\alpha},{ }^{\mathrm{t}} \mathcal{H}_{\alpha}$ and ${ }^{\mathrm{t}} \mathcal{P}_{B}$ the formal transposes of $L_{\alpha}, \mathcal{H}_{\alpha}$ and $\mathcal{P}_{B}$ on $\mathcal{S}\left(\mathbb{H}_{n}\right)$, respectively, then by $(2.7)$,

$$
{ }^{\mathrm{t}} \mathcal{H}_{\alpha}{ }^{\mathrm{t}} L_{\alpha} f=\mathcal{P}_{B}{ }^{\mathrm{t}} Q_{\alpha} f, \quad f \in \mathcal{S}\left(\mathbb{H}_{n}\right),
$$

since ${ }^{\mathrm{t}} \mathcal{P}_{B}=\mathcal{P}_{B}$.

Denote by $B_{r}$ the set of all $(z, u) \in \mathbb{H}_{n}$ such that $|z| \leq r,|u| \leq r$. Applying Proposition 2.1 to the central variable $u$, keeping $z$ fixed, we see that there is an $r>0$ such that

$$
\|\varphi\|_{2} \leq C\left\|\chi_{B_{2 r}}\left(\mathcal{P}_{B} \varphi\right)\right\|_{2}
$$

for every $\varphi \in C_{0}^{\infty}\left(\mathbb{H}_{n}\right)$ supported in $B_{r}$. Replacing $\varphi$ by ${ }^{\mathrm{t}} Q_{\alpha} \varphi$, and applying (2.12), we get

$$
\left\|{ }^{\mathrm{t}} Q_{\alpha} \varphi\right\|_{2} \leq C\left\|\chi_{B_{2 r}}\left(\mathcal{P}_{B}{ }^{\mathrm{t}} Q_{\alpha} \varphi\right)\right\|_{2}=\left\|\chi_{B_{2 r}}\left({ }^{\mathrm{t}} \mathcal{H}_{\alpha}{ }^{\mathrm{t}} L_{\alpha} \varphi\right)\right\|_{2} .
$$

Consequently,

$$
\begin{aligned}
\left\|{ }^{\mathrm{t}} Q_{\alpha} \varphi\right\|_{2} & \leq C \sup _{x \in B_{2 r}}\left|\left({ }^{\mathrm{t}} \mathcal{H}_{\alpha}\left({ }^{\mathrm{t}} L_{\alpha} \varphi\right)\right)(x)\right| \\
& =C \sup _{x \in B_{2 r}}\left|\int_{\mathbb{H}_{n}} H_{\alpha}^{m}(y)\left({ }^{\mathrm{t}} L_{\alpha} \varphi\right)(x y) d y\right| \leq C \sup _{x \in B_{2 r}}\left\|{ }^{\mathrm{t}} L_{\alpha} \varphi(x \cdot)\right\|_{(k)}
\end{aligned}
$$

for some Sobolev norm $\|\cdot\|_{(k)}$. But, since for $x \in B_{r}$, all functions ${ }^{\mathrm{t}} L_{\alpha} \varphi(x \cdot)$ are supported in a fixed compact subset, we may find an elliptic, left- 
invariant differential operator $D$ such that

$$
\left\|^{\mathrm{t}} L_{\alpha} \varphi(x \cdot)\right\|_{(k)} \leq\left\|D\left[{ }^{\mathrm{t}} L_{\alpha} \varphi(x \cdot)\right]\right\|_{2}=\left\|\left(D^{\mathrm{t}} L_{\alpha}\right) \varphi(x \cdot)\right\|_{2}=\left\|D^{\mathrm{t}} L_{\alpha} \varphi\right\|_{2},
$$

hence

$$
\left\|^{\mathrm{t}} Q_{\alpha} \varphi\right\|_{2} \leq C\left\|D^{\mathrm{t}} L_{\alpha} \varphi\right\|_{2}
$$

whenever $\operatorname{supp} \varphi \subset B_{r}$.

The operator ${ }^{\mathrm{t}} Q_{\alpha}$ is a constant coefficient operator acting on the variable $u$ only, and thus has a fundamental solution. So, arguing as before we see that there exists a bi-invariant differential operator $R$ such that

$$
\|\varphi\|_{2} \leq C\left\|R^{\mathrm{t}} Q_{\alpha} \varphi\right\|_{2}
$$

if $\operatorname{supp} \varphi \subset B_{r}$. But, $R$ commutes with $D$ and ${ }^{\mathrm{t}} Q_{\alpha}$, and so we obtain

$$
\|\varphi\|_{2} \leq C\left\|R D^{\mathrm{t}} L_{\alpha} \varphi\right\|_{2} \leq\left\|{ }^{\mathrm{t}} L_{\alpha} \varphi\right\|_{(l)}
$$

for a suitable Sobolev norm $\|\cdot\|_{(l)}$. This implies that $L_{\alpha}$ is locally solvable (see e.g. [3]).

\section{References}

[1] M. Christ and G. Karadzhov, Local solvability for a class of partial differential operators with double characteristics, Math. Res. Lett. 3 (1996), 511-526.

[2] F. De Mari, M. M. Peloso and F. Ricci, Analysis of second order differential operators with complex coefficients on the Heisenberg group, J. Reine Angew. Math. 464 (1995), $67-96$.

[3] L. Hörmander, Linear Partial Differential Operators, Grundlehren Math. Wiss. 116, Springer, 1976.

[4] D. Müller, M. M. Peloso and F. Ricci, On the solvability of homogeneous left-invariant differential operators on the Heisenberg group, J. Funct. Anal. 148 (1997), 368-383.

[5] - , - - - On local solvability for complex coefficient differential operators on the Heisenberg group, J. Reine Angew. Math. 513 (1999), 181-234.

[6] D. Müller and F. Ricci, Solvability for a class of non-homogeneous differential operators on two-step nilpotent groups, Math. Ann. 304 (1996), 517-547.

[7] D. Müller and C. Thiele, Normal forms of involutive complex Hamiltonian matrices under the real symplectic group, J. Reine Angew. Math. 513 (1999), 97-114.

[8] D. Müller and Z. Q. Zhang, Local solvability for positive combinations of generalized sub-Laplacians on the Heisenberg group, Proc. Amer. Math. Soc., to appear.

[9] E. M. Stein, An example on the Heisenberg group related to the Lewy operator, Invent. Math. 69 (1982), 209-216.

Mathematisches Seminar

Christian-Albrechts-Universität Kiel

Ludewig-Meyn-Str. 4

24098 Kiel, Germany

E-mail: mueller@math.uni-kiel.de
Department of Mathematics Tianjin University 300072 Tianjin, P.R. China E-mail: zqzhang@public.tpt.tj.cn 